\title{
Good Contacts for Quantitative In-Situ TEM Biasing Experiments with Movable Probes
}

\author{
Julio A. Rodríguez Manzo ${ }^{1}$, Daan Hein Alsem ${ }^{1}$, Norman J. Salmon ${ }^{1}$ and David Cooper ${ }^{2}$ \\ 1. Hummingbird Scientific, Lacey, USA. \\ 2. CEA, LETI, Minatec Campus, Grenoble, France.
}

With purpose-designed in-situ TEM sample holders it is possible to contact a sample inside an electron microscope with a movable probe. If the sample and probe are biased at different electrical potentials, contacting the sample with the movable probe creates an electrical circuit (Figure 1). This in-situ transmission electron microscope (TEM) platform allows to investigate the electrical properties of small contacts in combination with their structural and chemical state, by using all the battery of characterization techniques (high-resolution imaging, electron-based spectroscopy, electron diffraction, etc.) available in TEM. This approach has been used to investigate, for example, the switching of oxide resistive memories [1], anode materials for batteries [2,3], and the conductance through rows of atoms $[4,5]$.

Obtaining reliable quantitative information from in-situ biasing microscopy experiments that can be compared with "bulk" experiments is challenging. Standards are lacking and it's not unusual to have contradictory results about the same sample from different experiments. In this context, here we concentrate on the practical aspects of: 1) approaching the sample with coarse and fine movements and 2) preparing the movable probe, inside the TEM column, to obtain a good electrical contact between the sample and the probe prior to the experiment-by "good" we mean a contact bare of contamination or oxide layers that mask the intrinsic electrical behavior of the contact.

We will show experiments performed with a new in-situ TEM biasing sample holder that allows to approach the sample with the movable probe-usually a scanning tunneling microscopy (STM) tip -in manual coarse mode with smooth and uncoupled steps of $\mu \mathrm{m}$ or fractions of $\mu \mathrm{m}$ on the three spatial axes. Likewise, this holder allows to do the final contact approach (Figure 2) with a piezoelectric controlled mechanism that allows uncoupled nanometer-sized (or less) steps on the three spatial axes. We will compare and discuss the differences obtained with a standard diode junction when the biasing probe is clean and uncleaned, and provide details of how to clean the probe by passing a relative large current between the probe and the grid sustaining the sample, which is usually made of copper. Overlooking this cleaning step can affect the results of an in-situ TEM biasing experiment. This example illustrates that practical considerations can have a large impact on the outcome of the experiment and controlling these aspects is key to acquiring consistent data from in-situ microscopy experiments.

\section{References:}

[1] David Cooper et al, Advanced Materials 29 (2017), p. 1700212.

[2] Fei-Hu et al, ACS Nano 11 (2017), p. 8628.

[3] Ze Yang et al, Energy Storage Materials 9 (2017), p. 214.

[4] Hideaki Ohnishi, Yukihito Kondo and Kunio Takayanagi. Nature 395 (1998), p. 780.

[5] Ovidiu Cretu et al, Nano Letters 13 (2013), p. 3487. 

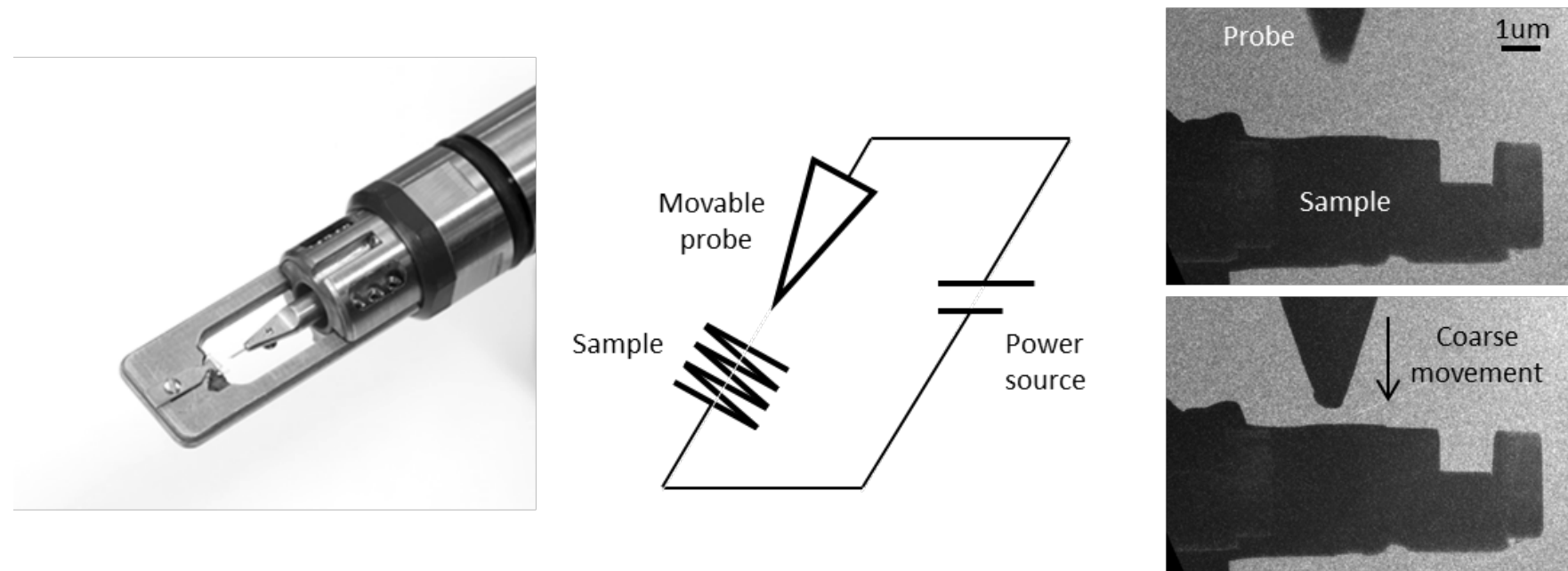

Figure 1. Detail of in-situ TEM biasing sample holder with a movable probe. From left to right, tip of in-situ TEM biasing sample holder, detailed circuit diagram, and probe coarse movement approaching a p-n junction sample fabricated in a FIB.

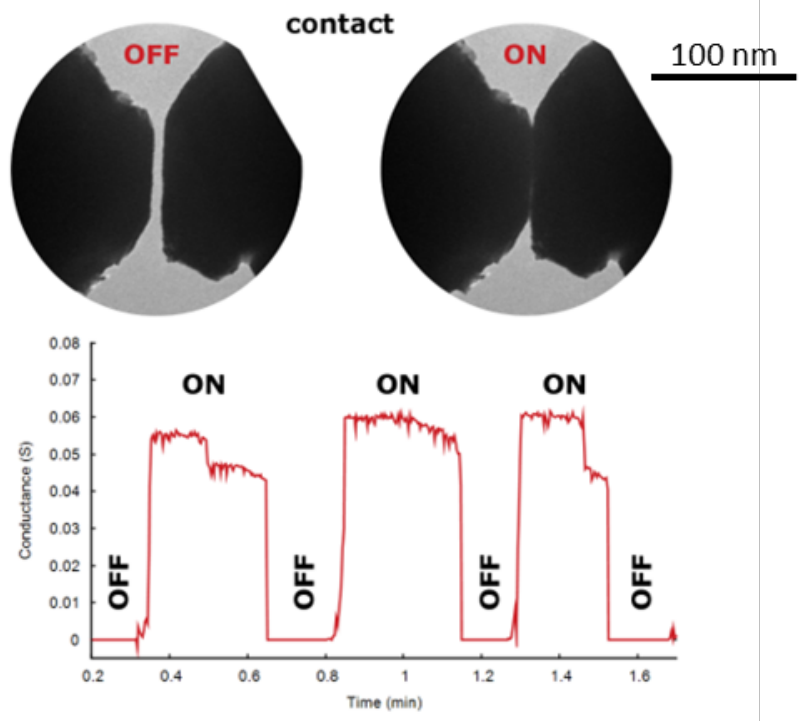

Figure 2. Conductance vs time plot, showing the conductance between two STM probes (Pt/Ir) as they connect and disconnect continually with the fine piezoelectrally-controlled movement inside a TEM. Contacting two STM probes requires high mechanical precision. The magnitude of the conductance for these contacts is about $10^{-2} \mathrm{~S}\left(10^{2} \Omega\right)$. To put things in perspective, the quantum of conductance is $7.7 \times$ $10^{-5} \mathrm{~S}(13 \mathrm{k} \Omega)$. The quantum of conductance is the unit of choice for people looking at nanocontacts and single-molecule transport. 